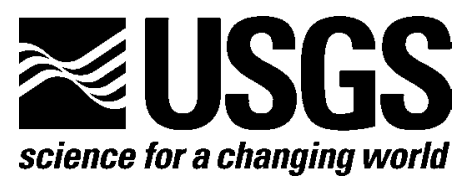

\title{
Compilation of Disruptions to Airports by Volcanic Activity (Version 1.0, 1944-2006)
}

By Marianne Guffanti, Gari C. Mayberry, Thomas J. Casadevall, and Richard Wunderman

Open-File Report 2007-1256

U.S. Department of the Interior

U.S. Geological Survey 


\section{U.S. Department of the Interior DIRK KEMPTHORNE, Secretary}

\section{U.S. Geological Survey Mark D. Myers, Director}

U.S. Geological Survey, Reston, Virginia: 2007

For product and ordering information:

World Wide Web: http://www.usgs.gov/pubprod

Telephone: 1-888-ASK-USGS

For more information on the USGS-the Federal source for science about the Earth, its natural and living resources, natural hazards, and the environment:

World Wide Web: http://www.usgs.gov

Telephone: 1-888-ASK-USGS

Suggested citation:

Guffanti, Marianne, Mayberry, G.C., Casadevall, T.J., and Wunderman, Richard, 2008, Compilation of disruptions to airports from volcanic activity (version 1.0, 1944-2006): U.S. Geological

Survey Open-File Report 2007-1256, 31 p. Available online at http://pubs.usgs.gov/of/2007/1256/.

Any use of trade, product, or firm names is for descriptive purposes only and does not imply endorsement by the U.S. Government.

Although this report is in the public domain, permission must be secured from the individual copyright owners to reproduce any copyrighted material contained within this report. 


\section{Contents}

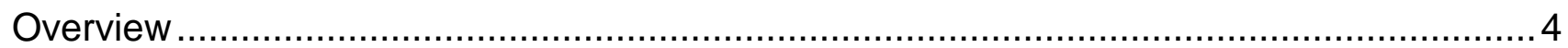

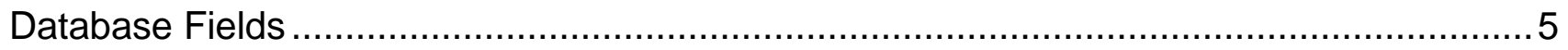

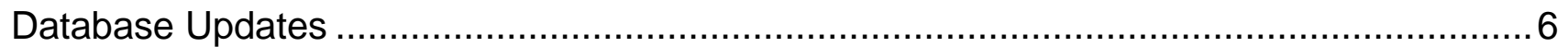

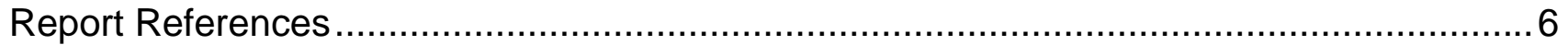

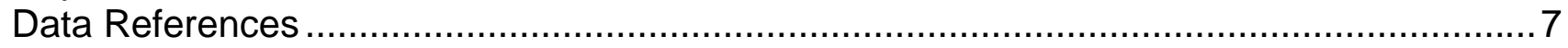

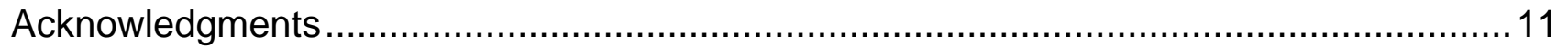

Tables

Table 1. Compilation of disruptions to airports by volcanic activity, 1944-2006.........12-26 


\section{Compilation of Disruptions to Airports by Volcanic Activity (Version 1.0, 1944-2006)}

By Marianne Guffanti, ${ }^{1}$ Gari C. Mayberry, ${ }^{2}$ Thomas J. Casadevall, ${ }^{3}$ and Rick Wunderman $^{4}$

${ }^{1}$ U.S. Geological Survey, Reston, Virginia

2 U.S. Geological Survey, Washington, D.C.

${ }^{3}$ U.S. Geological Survey, Denver, Colorado

${ }^{4}$ Smithsonian Institution, Washington, D.C.

\section{Overview}

Volcanic activity has caused significant hazards to numerous airports worldwide, with local to far-ranging effects on travelers and commerce (Guffanti and others, 2004; Casadevall, 1993). To more fully characterize the nature and scope of volcanic hazards to airports, we collected data on incidents of airports throughout the world that have been affected by volcanic activity, beginning in 1944 with the first documented instance of damage to modern aircraft and facilities in Naples, Italy, and extending through 2006. Information was gleaned from various sources, including news outlets, volcanological reports (particularly the Smithsonian Institution's Bulletin of the Global Volcanism Network), and previous publications on the topic (see Data References).

This report presents the full compilation of the data collected. For each incident, information about the affected airport and the volcanic source has been compiled as a record in a Microsoft Access database. The database is incomplete in so far as incidents may not have not been reported or documented, but it does present a good sample from diverse parts of the world. Not included are en-route diversions to avoid airborne ash clouds at cruise altitudes. For publication, the database has been converted to a Microsoft Excel spreadsheet (Table 1), a format with which most users are likely to be familiar.

Analysis of the database reveals that, at a minimum, 101 airports in 28 countries were impacted on 171 occasions from 1944 through 2006 by eruptions at 46 volcanoes. The number 
of affected airports (101) probably is better constrained than the number of incidents (171) because recurring disruptions at a given airport may have been lumped together or not reported by news agencies, whereas the initial disruption likely is noticed and reported and thus the airport correctly counted.

The types of hazardous volcanic activity that have affected airports are ashfall, ash in airspace around airports, lava flows, pyroclastic flows, and phreatic explosions. The primary hazard to airports is ashfall, which can cause loss of visibility, create slippery runways, infiltrate communication and electrical systems, interrupt ground services, and damage buildings and parked airplanes. Large amounts of ashfall are not necessary to disrupt operations at airports; temporary airport closures have resulted from accumulation of as little as a few millimeters of ash.

The effects of volcanic activity on airports include disruption of operations, damage to aircraft, and damage to facilities. The most common effect is temporary operational disruption, ranging from flight cancellations to airport closures for hours to weeks. Rarely, buildings, runways, and other physical infrastructure are destroyed or airports permanently closed. The risks are not restricted to airports located close to volcanoes, but can affect airports many hundreds of kilometers away. The size of affected airports varies from major international airports handling thousands of passengers and substantial cargo tonnages per day to regional airfields that, while much smaller, nevertheless are critical transportation infrastructure in some countries.

More detailed analysis of the database and discussion of methods to mitigate the adverse effects of volcanic activity on airports are presented in Guffanti and others (2008).

\section{Database Fields}

Incident \#: $\quad$ Unique identifier for each incident, assigned in this study

City/Airport: $\quad$ City, town, or military base in which airport is located/airport name

Country1: $\quad$ Country in which the airport is located

APLat: $\quad$ Airport latitude in decimal degrees; negative values indicate location in the southern hemisphere

APLong: $\quad$ Airport longitude in decimal degrees; negative values indicate location west of Prime Meridian (which runs through Greenwich, England)

Dist, km: $\quad$ Distance between location of airport and location of source volcano, calculated in this study using APLat, APLong, VLat, and VLong

Erupt Date Y/M/D: Date, as year/month/day, of eruption that caused the airport incident 
VEI:

Volcano:

VNum:

VLat:

VLong:

Country2:

Comment:

Hazard:

Days closed:

References:
Volcanic Explosivity Index (VEI) of eruptive activity that affected an airport, generally taken from the Smithsonian Institution's online global volcanism database which can be queried at http://www.volcano.si.edu/

Name of volcano that caused the airport incident (source volcano)

Unique identifying number assigned to each of the world's volcanoes by the Smithsonian Institution's Global Volcanism Program.

Volcano latitude in decimal degrees; negative values indicate location in the southern hemisphere

Volcano longitude in decimal degrees; negative values indicate location west of Prime Meridian (which runs through Greenwich, England)

Country in which the source volcano is located

Brief summary of the effects on the airport

Type of hazard at airport - AF, ashfall; AA, airborne ash; PF, pyroclastic flow; LF, lava flow; EX, phreatic or magmatic explosion; GD, ground deformation (inflation, subsidence, faulting).

Number of days (if known) an airport may have been closed

\section{Database Updates}

This report, published only as an online document, will be updated in future years. The compilation for the years 1944 through 2006 is identified in the report title as version 1.0. In future updates, the Open-File Report number will remain the same, while the version number will change to indicate the revised time period for which data is compiled. If any reader knows of inaccuracies in the data or has additional data and information to contribute, please contact the first author at guffanti@usgs.gov.

\section{Report References}

Casadevall, Thomas J., 1993, Volcanic ash and airports - discussion and recommendations from the workshop on impacts of volcanic ash on airport facilities: U.S. Geological Survey OpenFile Report 93-518, 53 p.

Guffanti, Marianne, Mayberry, G. C., Wunderman, Richard, and Casadevall, T. J., 2004, Effects of volcanic activity on airports, in Proceedings of the 2nd International Conference on 
Volcanic Ash and Aviation Safety, June 21-24, 2004, Alexandria, Virginia: Office of the Federal Coordinator for Meteorological Services and Supporting Research, session 5 p. 7-10, online at http://www.ofcm.noaa.gov/ICVAAS/Proceedings2004/ICVAAS2004-

Proceedings.htm/

Guffanti, Marianne, Mayberry, G.C., Casadevall, T.J., and Wunderman, R., 2008, Volcanic hazards to airports: Natural Hazards, Special Issue on Aviation Hazards from Volcanoes edited by Fred Prata and Andrew Tupper. DOI:10.1007/s11069-008-9254-2

\section{Data References}

\section{Bulletin of the Global Volcanism Network (BGVN)}

Bulletins are available online at http://www.volcano.si.edu/reports/bulletin/index.cfm/

Prior to 1986, some issues of BGVN were known as the Scientific Event Alert Network (SEAN)

$1982(07: 03)$

1986 (11:03)

1991 (16:04)

1991 (16:08)

1991 (16:10)

1992 (17:07)

1993 (18:05)

1994 (19:04)

1994 (19:08)

1995 (20:03)

1995 (20:10)

1996 (21:05)

1996 (21:09)

1997 (22:03)

1997 (22:06)

1997 (22:07)

1997 (22:09)

$1998(23: 05)$

1998 (23:11)

1998 (23:12)

1999 (24:02)

1999 (24:09)

1999 (24:10)

2000 (25:04)

2000 (25:06)

2000 (25:07)

2001 (26:07)

2001 (26:08)

2001 (26:09)

2001 (26:11)

2001 (26:12) 
$2002(27: 03)$

$2002(27: 04)$

$2002(27: 11)$

$2002(27: 12)$

$2003(28: 03)$

$2003(28: 05)$

$2003(28: 11)$

2004 (29:05)

$2004(29: 10)$

$2005(30: 02)$

$2005(30: 04)$

$2005(30: 05)$

$2005(30: 06)$

2005 (30:07)

SEAN 1983 (08:07)

SEAN 1983 (08:09)

SEAN $1983(08: 10)$

SEAN 1984 (09:05)

SEAN 1986 (11:09)

\section{News Reports}

Agence France Presse 3July2003

Agenzia Giornalistica Italia 6Dec2006

Airline Industry Information 25Feb2000

Associated Press 13July2003

Associated Press 22May2006

Australian Broadcasting Corp 11Oct2006

Diario del Hoy 17July2006

El Comercio 29Aug2003

El Comercio 18July2006

El Tiempo 24Nov2005

Fairchild Air Force Base Fact Sheet "Fairchild AFB History"

Jakarta Post 15Sept2004

Inquirer News Service 3May2005

LA Times Wire Reports 31May2005

New York Times 25Apr1944

Pacific Daily News 2Aug2005

Post Courier 2Mar2005

Prensa Libre 17Jan2000

Reuters 17April2005

Reuters 31July2001

Reuters 3Nov2002

Reuters 8June2004

Reuters 9Aug2002

Saipan Tribune 15Feb2005

Saipan Tribune 24May2003 
Saipan Tribune 8Aug2005

Times Online 5Nov2004

Stars\&Stripes 21Sept2004

\section{Published Articles}

Blong, R. J., 1984, Volcanic hazards: a sourcebook on the effects of eruptions: Academic Press Australia, Sydney, 424 p.

Casadevall, T. J., 1993, Volcanic ash and airports: discussion and recommendations from the workshop on impacts of volcanic ash on airport facilities: U. S. Geological Survey OpenFile Report 93-518, 53 p.

Casadevall, T. J., Delos Reyes, P. J., and Schneider, D. J.,1996, The 1991 Pinatubo eruptions and their effects on aircraft operations, in Newhall, C. G. and Punongbayan , R. S., eds., Fire and Mud - Eruptions and Lahars of Mount Pinatubo, Philippines. University of Washington Press, Seattle, p. 1071-1085.

Coltelli, M., 2006, Etna explosive eruptions and the INGV response to improve an ash-cloud monitoring system: Abstracts of the Fourth Conference Cities on Volcanoes, International Association of Volcanology and Chemistry of the Earth's Interior, Quito, Ecuador, 23-27 January 2006, p. 19.

Corwin, G., and Foster, H. L., 1959, The 1957 explosive eruption of Iwo-jima, Volcano Islands: American Journal of Science, v. 257, p. 161-171.

Guest, J., Murray, J., Kilburn, C., and Lopes, R., 1980, Eruptions of Mount Etna during 1979: Earthquake Information Bulletin, v. 2, no. 4, p. 154-160.

Guffanti, Marianne, Mayberry, G.C., Casadevall, T.J., and Wunderman, R., (2008), Volcanic hazards to airports: Natural Hazards, Special Issue on Aviation Hazards from Volcanoes, F. Prata and A Tupper (eds.), in press..

Jennings, P., 1969, Disruptions of the environmental balance: the eruptions of Mt. Agung and Mt. Kelut, Indonesia: University of Hawaii, Master of Arts thesis in Geography, 159 p.

Johnston, D. M., Houghton, B. F., Neall, V. E., Ronan, K. R., Paton, D., 2000, Impacts of the 1945 and 1995-1996 Ruapehu eruptions, New Zealand - an example of increasing societal vulnerability: Geological Society of America Bulletin, v. 112, p. 720-726.

Juhle, W., and Coulter, H., 1955, The Mt. Spurr eruption, July 9, 1953: Eos, Transactions of the American Geophysical Union, v. 36, p. 199-202.

Kienle, J., and Swanson, S. E., 1985, Volcanic hazards from future eruptions of Augustine volcano, Alaska: UAG R-275, University of Alaska, Fairbanks, 122 p.

Lloyd, A. T., 1990, Vulcan’s blast: Boeing Airliner, Seattle, p. 15-21. 
Schuster, R. L., 1981, Effects of the eruptions on civil works and operations in the Pacific Northwest, in Lipman, P. W., and Mullineaux, D. R., eds., The 1980 eruptions of Mount St. Helens: U. S. Geological Survey Professional Paper 1250, p. 701-718.

Schuster, R. L., 1983, Engineering aspects of the 1980 Mount St. Helens eruptions: Bulletin of the Assocation of Engineering Geologists, v. 20, p. 125-143.

Suryo, I., 1981, Report on the volcanic activity in Indonesia during the period 1961-1963: Bulletin of the Volcanological Survey of Indonesia, no. 104, 116 p.

Taylor, G. A., 1958, The 1951 eruption of Mount Lamington, Papua New Guinea: Bureau of Mineral Resources Bulletin 38, 117 p.

Tupper, A., Kamada, Y., Todo, N., and Miller, E., 2004, Aircraft encounters from the 18 August 2000 eruption at Miyake-jima, Japan, in Proceedings of the Second International Conference on Volcanic Ash and Aviation Safety, 21-24 June 2004, Alexandria, Virginia: Office of the Federal Coordinator for Meteorological Services and Supporting Research, session 1, p. 5-9.

Warrick, R. A., Anderson, J., Downing, T., Lyons, J., Ressler, J., Warrick, M., and Warrick T., 1981, Four communities under ash after Mount St. Helens: Program on Technology, Environment, and Man, Monograph 34, Institute of Behavioral Science, Univ. of Colorado, $143 \mathrm{p}$.

Wilcox , R. E., 1959, Some effects of recent volcanic ash falls with special reference to Alaska: U. S. Geological Survey Bulletin 1028-N, p. 409-476.

\section{Unpublished Sources}

W. Aspinall, pers. comm. 2003

AFWA Alert 16Sept2004 (Air Force Weather Agency)

E. Duarte pers. comm. 2006

S. Eckhert pers. comm. 2002

J. Ewert pers. comm. 2002

C. Holliday pers. comm. 2002, 2006

D. Innes pers. comm. 2003, 2006

S. Karlsdottir pers. comm. 2006

P. Lechner written comm. 2004

C. Neal pers. comm. 2006

C. Newhall pers. comm.. 2007

L. Salinas pers. comm. 2003

R. Stewart pers. comm. 2005

VSI written comm. 2004 (Volcanological Survey of Indonesia) 


\section{Acknowledgments}

This work was supported by the USGS Volcano Hazards Program. Chris Newhall and Steve Brantley provided helpful reviews of this report. We thank the following people for providing data and fact-checking entries: Mauro Coltelli, John Ewert, Chris Harpel, Charles Holliday, Tony Hurst, David Innes, Tina Neal, Herman Patia, Brad Scott, Sally Kuhn Sennert, Rod Stewart, and Grace Swanson. 
Table 1. Compilation of Disruptions to Airports by Volcanic Activity (Version 1.0, 1944-2006)

By M. Guffanti, G.C. Mayberry, T.J. Casadevall, R. Wunderman. Data fields explained in report text. (AP=Airport)

\begin{tabular}{|c|c|c|c|c|c|c|c|c|c|c|c|}
\hline Incident \# & CitylAirport & Country1 & APLat & APLong & Dist, km & Erupt Date Y/MVD & VE & Volcano & VNum & VLat & VLong \\
\hline 2006AP-5.2 & Catania/Sigonella Naval Air Station & Italy (USN base) & 37.40 & 14.92 & 38 & $2006 / 11 / 24-12 / 16$ & $2 ?$ & Etna & 0101-06 & 37.734 & 15.004 \\
\hline 2006AP-5.1 & Catania/Fontanarossa Intl AP & Italy & 37.467 & 15.067 & 30 & $2006 / 11 / 24-12 / 16$ & $2 ?$ & Etna & 0101-06 & 37.734 & 15.004 \\
\hline 2006AP-4.1 & Kokopo/Tokua AP & Papua New Guinea & -4.340 & 152.380 & 21 & $2006 / 10 / 07-10$ & 3 & Rabaul & 0502-14 & -4.271 & 152.203 \\
\hline 2006AP-3.6 & Manta/Manta AP & Ecuador (US base) & -0.95 & -80.683 & 256 & $2006 / 08 / 16-17$ & 4 & Tungurahua & $1502-08$ & -1.467 & -78.442 \\
\hline 2006AP-3.5 & Riobamba/Riobamba AP & Ecuador & -1.40 & -78.38 & 10 & 2006/08/16-17 & 4 & Tungurahua & 1502-08 & -1.467 & -78.442 \\
\hline 2006AP-3.4 & Latacunga/Cotopaxi Intl AP & Ecuador & 0.933 & -78.62 & 268 & $2006 / 08 / 16-17$ & 4 & Tungurahua & $1502-08$ & -1.467 & -78.442 \\
\hline 2006AP-3.3 & Ambato/Ambato AP & Ecuador & -1.2 & -78.57 & 33 & $2006 / 08 / 16-17$ & 4 & Tungurahua & $1502-08$ & -1.467 & -78.442 \\
\hline 2006AP-3.2 & Quito/Mariscal Sucre Intl AP & Ecuador & -0.133 & -78.483 & 148 & $2006 / 08 / 16-17$ & 4 & Tungurahua & 1502-08 & -1.467 & -78.442 \\
\hline 2006AP-3.1 & Guayaquil/Simon Bolvar AP & Ecuador & -2.167 & -79.867 & 177 & $2006 / 08 / 16-17$ & 4 & Tungurahua & $1502-08$ & -1.467 & -78.442 \\
\hline 2006AP-2.5 & Caracas/Ciudad Bolivar AP & Venezuela & 8.13 & -68.53 & 1175 & $2006 / 05 / 20-21$ & 4 & Soufriere Hills & $1600-05$ & 16.72 & -62.18 \\
\hline 2006AP-2.4 & Oranjestad/Queen Beatrix Intl AP & Netherlands (Aruba) & 12.5 & -70.012 & 965 & $2006 / 05 / 20-21$ & 4 & Soufriere Hills & $1600-05$ & 16.72 & -62.18 \\
\hline 2006AP-2.3 & Curacao/Hato AP & Netherlands Antilles & 12.2 & -68.97 & 887 & $2006 / 05 / 20-21$ & 4 & Soufriere Hills & $1600-05$ & 16.72 & -62.18 \\
\hline 2006AP-2.2 & Havana/Jose Marti Intl AP & Cuba & 23.02 & -82.38 & 2224 & $2006 / 05 / 20-21$ & 4 & Soufriere Hills & $1600-05$ & 16.72 & -62.18 \\
\hline 2006AP-2.1 & Santo Domingo/AP de las Americas & Dominican Republic & 18.47 & -69.9 & 841 & 2006/05/20-21 & 4 & Soufriere Hills & $1600-05$ & 16.72 & -62.18 \\
\hline 2006AP-1.1 & Anchorage/Anchorage Intl AP & USA & 61.167 & -149.983 & 275 & 2006/01/13-17 & 1 & Augustine & $1103-01$ & 59.37 & -153.42 \\
\hline 2005AP-9.1 & Pasto/Pasto Cano AP & Colombia & 1.467 & -77.25 & 31 & $2005 / 11 / 24$ & 2 & Galeras & 1501-08 & 1.22 & -77.37 \\
\hline 2005AP-8.1 & Saipan/Saipan Intl AP & USA (CNMI) & 15.13 & 145.7 & 136 & 2005/08/01-05 & 1 & Anatahan & $0804-20$ & 16.35 & 145.67 \\
\hline 2005AP-7.1 & Colima/Colima AP & Mexico & 19.3 & -103.667 & 24 & $2005 / 05 / 31$ & 3 & Colima & 1401-04 & 19.514 & -103.62 \\
\hline 2005AP-6.1 & Kalibo/Kalibo AP & Philippines & 11.7 & 122.37 & 166 & $2005 / 05 / 03$ & 1 & Canlaon & $0702-02$ & 10.412 & 123.132 \\
\hline 2005AP-5.1 & Moroni/Hahaya-Iconi AP & Comoros & -11.7 & 43.233 & 17 & 2005/04/16-18 & 3 & Karthala & 0303-01 & -11.75 & 43.38 \\
\hline 2005AP-4.1 & Saipar/Saipan Intl AP & USA (CNMI) & 15.13 & 145.7 & 136 & $2005 / 04 / 06$ & 3 & Anatahan & 0804-20 & 16.35 & 145.67 \\
\hline 2005AP-3.1 & Kokopo/Tokua AP & Papua New Guinea & -4.340 & 152.380 & 21 & $2005 / 02 / 22-24$ & 2 & Rabaul & 0502-14 & -4.271 & 152.203 \\
\hline 2005AP-2.1 & Saipan/Saipan Intl AP & USA (CNMI) & 15.13 & 145.7 & 136 & 2005/02/14 & 1 & Anatahan & 0804-20 & 16.35 & 145.67 \\
\hline 2005AP-1.1 & Kokopo/Tokua AP & Papua New Guinea & -4.340 & 152.380 & 33 & $2005 / 01 / 27$ & 4 & Manam & 0501-02 & -4.1 & 145.061 \\
\hline 2004AP-6.1 & Reykjavik/Keflavik AP & Iceland & 63.983 & -22.6 & 260 & 2004/11/01-04 & 4 & Grimsvotn & 1703-01 & 64.42 & -17.33 \\
\hline 2004AP-5.1 & Boram / Wewak AP & Papua New Guinea & -3.598 & 143.667 & 950 & $2004 / 10 / 24$ & 4 & Manam & 0501-02 & -4.08 & 145.037 \\
\hline 2004AP-4.1 & Yokota Air Base & Japan (US base) & 35.75 & 139.35 & 103 & 2004/09/13-16 & 2 & Asama & 0803-11 & 36.4 & 138.53 \\
\hline 2004AP-3.1 & Maumere/Waioty AP & Indonesia & -8.633 & 122.25 & 22 & 2004/09/06? & 2 & Egon & 0604-16 & -8.67 & 122.45 \\
\hline 2004AP-2.1 & Sangihe/Tahuna AP & Indonesia & -1.183 & 123.817 & 571 & 2004/06/08-09 & 2 & Amu & 0607-04 & 3.67 & 125.50 \\
\hline 2004AP-1.1 & Ust-Kamchatsk & Russia & unknown & unknown & & $2004 / 05 / 09$ & 4 & Sheveluch & $1000-27$ & 56.63 & 161.32 \\
\hline 2003AP-5.4 & Cuenca/Mariscal La Mar AP & Ecuador & -2.9 & -78.967 & 170 & $2003 / 08 / 27$ & 2 & Tungurahua & 1502-08 & -1.467 & -78.442 \\
\hline 2003AP-5.3 & Quito/Mariscal Sucre Intl AP & Ecuador & -0.133 & -78.483 & 148 & $2003 / 08 / 27$ & 2 & Tungurahua & $1502-08$ & -1.467 & -78.442 \\
\hline 2003AP-5.2 & Ambato/Ambato AP & Ecuador & -1.2 & -78.57 & 33 & $2003 / 08 / 27$ & 2 & Tungurahua & $1502-08$ & -1.467 & -78.442 \\
\hline 2003AP-5.1 & Guayaquil/Simon Bolivar AP & Ecuador & -2.167 & -79.867 & 177 & $2003 / 08 / 27$ & 2 & Tungurahua & 1502-08 & -1.467 & -78.442 \\
\hline 2003AP-4.7 & St. Kitts/St. Kitts AP & St. Kitts & 17.2 & -62.7 & 77 & $2003 / 07 / 12-13$ & 3 & Soufriere Hills & $1600-05$ & 16.72 & -62.18 \\
\hline 2003AP-4.6 & Unspecified AP on Anguilla & UK (Anguilla) & $?$ & $?$ & & 2003/07/12-13 & 3 & Soufriere Hills & $1600-05$ & 16.72 & -62.18 \\
\hline 2003AP-4.5 & San Juan/Luis Munoz Marin Intl AP & USA (Puerto Rico) & 18.45 & -66 & 448 & $2003 / 07 / 12-13$ & 3 & Soufriere Hills & $1600-05$ & 16.72 & -62.18 \\
\hline 2003AP-4.4 & St. Thomas/Cyril King AP & USA (Virgin Islands) & 18.333 & -64.967 & 346 & $2003 / 07 / 12-13$ & 3 & Soufriere Hills & $1600-05$ & 16.72 & -62.18 \\
\hline
\end{tabular}


Table 1. Compilation of Disruptions to Airports by Volcanic Activity (Version 1.0, 1944-2006)

By M. Guffanti, G.C. Mayberry, T.J. Casadevall, R. Wunderman. Data fields explained in report text. (AP=Airport)

\begin{tabular}{|c|c|c|c|c|c|c|c|c|c|c|c|}
\hline Incident \# & CitylAirport & Country1 & APLat & APLong & Dist, km & Erupt Date Y/MVD & VE & Volcano & VNum & VLat & VLong \\
\hline 2003AP-4.3 & Sint Maarten/Princess Juliana Intl AP & Netherlands Antilles & 18.05 & -63.117 & 178 & 2003/07/12-13 & 3 & Soufriere Hills & $1600-05$ & 16.72 & -62.18 \\
\hline 2003AP-4.2 & Unspecified AP on Guadeloupe & France (Guadeloupe) & unknown & unknown & & 2003/07/12-13 & 3 & Soufriere Hills & $1600-05$ & 16.72 & -62.18 \\
\hline 2003AP-4.1 & Roseau/Cane Field AP & Dominica & 15.5 & -61.3 & 165 & $2003 / 07 / 12-13$ & 3 & Soufriere Hills & $1600-05$ & 16.72 & -62.18 \\
\hline 2003AP-3.2 & Guam/Guam Intl AP & USA (Guam) & 13.4 & 144.73 & 343 & $2003 / 05 / 23$ & 3 & Anatahan & 0804-20 & 16.35 & 145.67 \\
\hline 2003AP-3.1 & Saipan/Saipan Intl AP & USA (CNMI) & 15.13 & 145.7 & 136 & $2003 / 05 / 23$ & 3 & Anatahan & 0804-20 & 16.35 & 145.67 \\
\hline 2003AP-2.1 & San Juan/Luis Munoz Marin Intl AP & USA (Puerto Rico) & 18.45 & -66 & 448 & $2003 / 03 / 17$ & 3 & Soufriere Hills & $1600-05$ & 16.72 & -62.18 \\
\hline 2003AP-1.1 & Kokopo/Tokua AP & Papua New Guinea & -4.340 & 152.380 & 21 & 2003/01/01-31 & 2 & Rabaul & 0502-14 & -4.271 & 152.203 \\
\hline 2002AP-6.1 & Quito/Mariscal Sucre Intl AP & Ecuador & -0.133 & -78.483 & 92 & $2002 / 11 / 03$ & 4 & Reventador & 1502-01 & -0.078 & -77.656 \\
\hline 2002AP-5.3 & Catania/Fontanorossa Intl AP & Italy & 37.067 & 15.067 & 30 & $2002 / 10 / 27-2003 / 01 / 28$ & 3 & Etna & 0101-06 & 37.734 & 15.004 \\
\hline 2002AP-5.2 & Reggio di Calabria/ Reggio di Calabria AP & Italy & 38.067 & 15.65 & 68 & $2002 / 10 / 27-2003 / 01 / 28$ & 3 & Etna & 0101-06 & 37.734 & 15.004 \\
\hline 2002AP-5.1 & Catania/Sigonella Naval Air Station & Italy (USN base) & 37.40 & 14.92 & 38 & 2002/10/27-2003/01/28 & 3 & Etna & 0101-06 & 37.734 & 15.004 \\
\hline 2002AP-4.1 & Kokopo/Tokua AP & Papua New Guinea & -4.340 & 152.380 & 21 & $2003 / 10 / 20+$ & 2 & Rabaul & 0502-14 & -4.271 & 152.203 \\
\hline 2002AP-3.1 & Roosevelt Roads NAS & USA (Puerto Rico) & 18.26 & -65.64 & 405 & $2002 / 09 / 30$ & 3 & Soufriere Hills & $1600-05$ & 16.72 & -62.18 \\
\hline 2002AP-2.1 & Kimbe/Hoskins AP & Papua New Guinea & -5.462 & 150.405 & 18 & $2002 / 08 / 05$ & 3 & Pago & 0502-08 & -5.58 & 150.52 \\
\hline 2002AP-1.1 & Goma/Goma AP & D.R. Congo & -1.667 & 29.233 & 16 & $2002 / 01 / 17-21$ & 1 & Nyiragongo & 0203-03 & -1.52 & 29.25 \\
\hline 2001AP-5.4 & St. Thomas/Cyril King AP & USA (Virgin Islands) & 18.333 & -64.967 & 346 & $2001 / 07 / 29$ & 3 & Soufriere Hills & $1600-05$ & 16.72 & -62.18 \\
\hline 2001AP-5.3 & San Juan/Luis Munoz Marin Intl AP & USA (Puerto Rico) & 18.45 & -66 & 449 & $2001 / 07 / 29$ & 3 & Soufriere Hills & $1600-05$ & 16.72 & -62.18 \\
\hline 2001AP-5.2 & St. Croix/Alex. Hamilton AP & USA (Virgin Islands) & 17.717 & -64.783 & 298 & $2001 / 07 / 29$ & 3 & Soufriere Hills & $1600-05$ & 16.72 & -62.18 \\
\hline 2001AP-5.1 & Sint Maarten/Princess Juliana Intl AP & Netherlands Antilles & 18.05 & -63.117 & 178 & $2001 / 07 / 29$ & 3 & Soufriere Hills & $1600-05$ & 16.72 & -62.18 \\
\hline 2001AP-4.1 & Catania/Fontanarossa Intl AP & Italy & 37.467 & 15.067 & 30 & 2001/07/21-30 & 3 & Etna & 0101-06 & 37.734 & 15.004 \\
\hline 2001AP-3.1 & Mexico City/Benito Juarez Intl AP & Mexico & 19.433 & -99.083 & 67 & $2001 / 07 / 03$ & 3 & Popcatepetl & 1401-09 & 19.023 & -98.622 \\
\hline 2001AP-2.1 & Legaspi/Legaspi AP & Philippines & 13.167 & 123.733 & 11 & $2001 / 06 / 23-29$ & 3 & Mayon & 0703-03 & 13.257 & 123.685 \\
\hline 2001AP-1.1 & Manado/Sam Ratulangi AP & Indonesia & 1.533 & 124.917 & 24 & $2001 / 05 / 20$ & 2 & Lokon & 0606-10 & 1.358 & 124.792 \\
\hline 2000AP-6.1 & Miyakejima/Miyakejima AP & Japan & 34.067 & 139.567 & 4 & $2000 / 08 / 18$ & 3 & Myake-jima & 0804-04 & 34.08 & 139.53 \\
\hline 2000AP-5.1 & Tauranga/Tauranga AP & New Zealand & -37.667 & 176.2 & 88 & 2000/07/19 & 3 & White Island & 0401-04 & -37.52 & 177.18 \\
\hline 2000AP-4.2 & Catania/Sigonella Naval Air Station & Italy (USN base) & 37.40 & 14.92 & 38 & $2000 / 06 / 05$ & 3 & Etna & 0101-06 & 37.734 & 15.004 \\
\hline 2000AP-4.1 & Catania/Fontanarossa Intl AP & Italy & 37.467 & 15.067 & 30 & 2000/06/05 & 3 & Etna & 0101-06 & 37.734 & 15.004 \\
\hline 2000AP-3.1 & Legaspi/Legaspi AP & Philippines & 13.167 & 123.733 & 11 & $2000 / 02 / 23-24$ & 3 & Mayon & 0703-03 & 13.257 & 123.685 \\
\hline 2000AP-2.2 & Riobamba/Riobamba AP & Ecuador & -1.40 & -78.38 & 10 & 2000/02/08 & 2 & Tungurahua & $1502-08$ & -1.467 & -78.442 \\
\hline 2000AP-2.1 & Ambato/Ambato AP & Ecuador & -1.2 & -78.567 & 33 & 2000/02/08 & 2 & Tungurahua & 1502-08 & -1.467 & -78.442 \\
\hline 2000AP-1.1 & Guatemala City/La Aurora Intl AP & Guatemala & 14.567 & -90.533 & 22 & 2000/01/16 & 1 & Pacaya & $1402-11$ & 14.381 & -90.601 \\
\hline 1999AP-3.1 & Quito/Mariscal Sucre Intl AP & Ecuador & -0.133 & -78.483 & 13 & 1999/11/24 & 3 & Pichincha & $1502-02$ & -0.171 & -78.598 \\
\hline 1999AP-2.1 & Quito/Mariscal Sucre Intl AP & Ecuador & -0.133 & -78.483 & 13 & 1999/10/05-07 & 3 & Pichincha & $1502-02$ & -0.171 & -78.598 \\
\hline 1999AP-1.1 & Guatemala City/La Aurora Intl AP & Guatemala & 14.567 & -90.533 & 39 & $1999 / 05 / 21$ & 2 & Fuego & $1402-09$ & 14.473 & -90.880 \\
\hline 1998AP-4.1 & Mexico City/Benito Juarez Intl AP & Mexico & 19.433 & -99.083 & 67 & 1998/12/20 & 3 & Popcatepetl & 1401-09 & 19.023 & -98.622 \\
\hline 1998AP-3.1 & Guatemala City/La Aurora Intl AP & Guatemala & 14.567 & -90.533 & 22 & 1998/09/18-19 & 3 & Pacaya & $1402-11$ & 14.381 & -90.601 \\
\hline 1998AP-2.1 & Catania/Fontanarossa Intl AP & Italy & 37.467 & 15.067 & 30 & 1998/07/22 & 3 & Etna & 0101-06 & 37.734 & 15.004 \\
\hline 1998AP-1.1 & Guatemala City/La Aurora Intl AP & Guatemala & 14.567 & -90.533 & 22 & $1998 / 05 / 20$ & 3 & Pacaya & $1402-11$ & 14.381 & -90.601 \\
\hline
\end{tabular}


Table 1. Compilation of Disruptions to Airports by Volcanic Activity (Version 1.0, 1944-2006)

By M. Guffanti, G.C. Mayberry, T.J. Casadevall, R. Wunderman. Data fields explained in report text. (AP=Airport)

\begin{tabular}{|c|c|c|c|c|c|c|c|c|c|c|c|}
\hline Incident \# & CitylAirport & Country1 & APLat & APLong & Dist, km & Erupt Date Y/MVD & VE & Volcano & VNum & VLat & VLong \\
\hline 1997AP-5.1 & Saint John's/V.C. Bird Intl AP & Antigua & 17.05 & -61.8 & 55 & 1997/09/07 & 3 & Soufriere Hills & $1600-05$ & 16.72 & -62.18 \\
\hline 1997AP-4.1 & Mexico City/Benito Juarez Intl AP & Mexico & 19.433 & -99.083 & 67 & 1997/06/30 & 3 & Popocatepetl & $1401-09$ & 19.023 & -98.622 \\
\hline 1997AP-3.1 & PlymouthW. H. Bramble AP & UK (Montserrat) & 16.732 & -62.177 & 1 & $1997 / 06 / 25$ & 3 & Soufriere Hills & $1600-05$ & 16.72 & -62.18 \\
\hline 1997AP-2.1 & PlymouthW. H. Bramble AP & UK (Montserrat) & 16.732 & -62.177 & 1 & 1997/06/17 & 3 & Soufriere Hills & $1600-05$ & 16.72 & -62.18 \\
\hline 1997AP-1.1 & Kokopo/Tokua AP & Papua New Guinea & -4.340 & 152.380 & 21 & 1997/03/14 & 2 & Rabaul & 0502-14 & -4.271 & 152.203 \\
\hline 1996AP-3.1 & Unspecified AP on Guadeloupe & France (Guadeloupe) & unknown & unknown & & 1996/09/17 & 3 & Soufriere Hills & $1600-05$ & 16.72 & -62.18 \\
\hline 1996AP-2.1 & PlymouthW. H. Bramble AP & UK (Montserrat) & 16.732 & -62.177 & 1 & 1996/09/02-03 & 3 & Soufriere Hills & $1600-05$ & 16.72 & -62.18 \\
\hline 1996AP-1.11 & Palmerston North/Palmerston N AP & New Zealand & -40.317 & 175.617 & 115 & 1996/06-08 & 3 & Ruapehu & 0401-10 & -39.28 & 175.57 \\
\hline 1996AP-1.10 & New Plymouth/New Plymouth AP & New Zealand & -39.033 & 174.183 & 123 & 1996/06-08 & 3 & Ruapehu & 0401-10 & -39.28 & 175.57 \\
\hline 1996AP-1.09 & Wanganui/Wanganui AP & New Zealand & -39.967 & 175.017 & 90 & 1996/06-08 & 3 & Ruapehu & 0401-10 & -39.28 & 175.57 \\
\hline 1996AP-1.08 & Gisborne/Gisborne AP & New Zealand & -38.67 & 177.98 & 219 & 35224 & 3 & Ruapehu & 0401-10 & -39.28 & 175.57 \\
\hline 1996AP-1.07 & Taupo/Taupo AP & New Zealand & -38.667 & 176.133 & 87 & 1996/06-08 & 3 & Ruapehu & 0401-10 & -39.28 & 175.57 \\
\hline 1996AP-1.06 & Napier/Hastings AP & New Zealand & -39.47 & 176.87 & 114 & 1996/06-08 & 3 & Ruapehu & 0401-10 & -39.28 & 175.57 \\
\hline 1996AP-1.05 & Whakatane/Mhakatane AP & New Zealand & -37.933 & 176.933 & 191 & 1996/06-08 & 3 & Ruapehu & 0401-10 & -39.28 & 175.57 \\
\hline 1996AP-1.04 & Hamilton/Hamilton AP & New Zealand & -37.867 & 175.133 & 159 & 1996/06-08 & 3 & Ruapehu & 0401-10 & -39.28 & 175.57 \\
\hline 1996AP-1.03 & Tauranga/Tauranga AP & New Zealand & -37.667 & 176.2 & 88 & 1996/06-08 & 3 & Ruapehu & 0401-10 & -39.28 & 175.57 \\
\hline 1996AP-1.02 & Rotorua/Rotorua AP & New Zealand & -38.15 & 176.27 & 120 & 1996/06/17 & 3 & Ruapehu & 0401-10 & -39.28 & 175.57 \\
\hline 1996AP-1.01 & Auckland/Auckland Intl AP & New Zealand & -36.867 & 174.767 & 277 & 1996/06/17 & 3 & Ruapehu & 0401-10 & -39.28 & 175.57 \\
\hline 1995AP-3.1 & PlymouthW. H. Bramble AP & UK (Montserrat) & 16.732 & -62.177 & 1 & $1995 / 10 / 21$ & 3 & Soufriere Hills & $1600-05$ & 16.72 & -62.18 \\
\hline 1995AP-2.9 & Palmerston North/Palmerston N AP & New Zealand & -40.317 & 175.617 & 115 & 1995/09-10 & 3 & Ruapehu & 0401-10 & -39.28 & 175.57 \\
\hline 1995AP-2.8 & Rotorua/Rotorua AP & New Zealand & -38.15 & 176.27 & 120 & $1995 / 09-10$ & 3 & Ruapehu & 0401-10 & -39.28 & 175.57 \\
\hline 1995AP-2.7 & Taupo/Taupo AP & New Zealand & -38.667 & 176.133 & 87 & 1995/09-10 & 3 & Ruapehu & 0401-10 & -39.28 & 175.57 \\
\hline 1995AP-2.6 & Wanganui/Wanganui AP & New Zealand & -39.967 & 175.017 & 90 & 1995/09-10 & 3 & Ruapehu & 0401-10 & -39.28 & 175.57 \\
\hline 1995AP-2.5 & Hamilton/Hamilton AP & New Zealand & -37.867 & 176.133 & 159 & $1995-09-10$ & 3 & Ruapehu & 0401-10 & -39.28 & 175.57 \\
\hline 1995AP-2.4 & Whakatane/Whakatane AP & New Zealand & -37.933 & 176.933 & 191 & 1995/09-10 & 3 & Ruapehu & $0401-10$ & -39.28 & 175.57 \\
\hline 1995AP-2.3 & Tauranga/Tauranga AP & New Zealand & -37.667 & 176.2 & 88 & $1995 / 09-10$ & 3 & Ruapehu & 0401-10 & -39.28 & 175.57 \\
\hline 1995AP-2.2 & Napier/Hastings AP & New Zealand & -39.47 & 176.87 & 114 & 1995/10/14 & 3 & Ruapehu & 0401-10 & -39.28 & 175.57 \\
\hline 1995AP-2.1 & Gisborne/Gisborne AP & New Zealand & -38.67 & 177.98 & 219 & 1995/10/11-12 & 3 & Ruapehu & $0401-10$ & -39.28 & 175.57 \\
\hline 1995AP-1.1 & Puebla/Puebla-Hermanos Serdan AP & Mexico & 19.135 & -98.368 & 29 & $1995 / 01 / 27$ & 2 & Popcatepetl & 1401-09 & 19.023 & -98.622 \\
\hline 1994AP-2.2 & Neuquen/Neuquen AP & Argentina & -38.95 & -68.133 & 313 & $1994 / 05 / 17$ & 2 & Laima & $1507-11$ & -38.692 & -71.729 \\
\hline 1994AP-1.2 & Rabaul/Rabaul AP & Papua New Guinea & -4.214 & 152.186 & 7 & 1994/09/19 & 4 & Rabaul & $0502-14$ & -4.271 & 152.203 \\
\hline 1994AP-1.1 & Kavieng/Kavieng AP & Papua New Guinea & -2.579 & 150.808 & 244 & $1994 / 09 / 19$ & 4 & Rabaul & 0502-14 & -4.271 & 152.203 \\
\hline 1993AP-2.1 & Pasto/Pasto Cano AP & Colombia & 1.467 & -77.25 & 31 & 1993/06/07 & 2 & Galeras & 1501-08 & 1.22 & -77.37 \\
\hline 1993AP-1.4 & Salta/Salta AP & Argentina & -24.85 & -65.483 & 281 & 1993/04/18-21 & 4 & Lascar & $1505-10$ & -23.37 & -67.73 \\
\hline 1993AP-1.3 & Cordoba/Cordoba AP & Argentina & -31.317 & -64.217 & 949 & $1993 / 04 / 18-21$ & 4 & Lascar & $1505-10$ & -23.37 & -67.73 \\
\hline 1993AP-1.2 & Asuncion/Presidente Gen Stroessner AP & Paraguay & -25.267 & -57.667 & 1041 & 1993/04/18-21 & 4 & Lascar & $1505-10$ & -23.37 & -67.73 \\
\hline 1993AP-1.1 & Jujuy/E Cadillal AP & Argentina & -24.233 & -65.267 & 268 & 1993/04/18-21 & 4 & Lascar & $1505-10$ & -23.37 & -67.73 \\
\hline 1992AP-2.4 & Kenai/Kenai Municipal AP & USA & 60.583 & -152.15 & 35 & 1992/08/18 & 4 & Spurr & $1103-04$ & 61.3 & -152.75 \\
\hline
\end{tabular}


Table 1. Compilation of Disruptions to Airports by Volcanic Activity (Version 1.0, 1944-2006)

By M. Guffanti, G.C. Mayberry, T.J. Casadevall, R. Wunderman. Data fields explained in report text. (AP=Airport)

\begin{tabular}{|c|c|c|c|c|c|c|c|c|c|c|c|}
\hline Incident \# & CitylAirport & Country1 & APLat & APLong & Dist, km & Erupt Date Y/MVD & $\mathbf{V E}$ & Volcano & VNum & VLat & VLong \\
\hline 1992AP-2.3 & Anchorage/Merrill Field & USA & 61.2 & -149.833 & 130 & $1992 / 08 / 18$ & 4 & Spurr & $1103-04$ & 61.30 & -152.25 \\
\hline 1992AP-2.2 & Anchorage/Emendorf AFB & USA & 61.253 & -149.792 & 131 & 1992/08/18 & 4 & Spurr & $1103-04$ & 61.30 & -152.25 \\
\hline 1992AP-2.1 & Anchorage/Anchorage Intl AP & USA & 61.167 & -149.983 & 122 & 1992/08/18 & 4 & Spurr & $1103-04$ & 61.30 & -152.25 \\
\hline 1992AP-1.1 & Kagoshima/Kagoshima AP & Japan & 31.55 & 130.567 & 10 & 1992/06/06 & 3 & Sakurajima & 0802-08 & 31.58 & 130.67 \\
\hline 1991AP-6.2 & Medan/Polonia AP & Indonesia & 3.567 & 98.683 & 2911 & 1991/10/24 & 3 & Lokon & 0606-10 & 1.358 & 124.792 \\
\hline 1991AP-6.1 & Manado/Sam Ratulangi AP & Indonesia & 1.533 & 124.917 & 24 & $1991 / 10 / 24$ & 3 & Lokon & 0606-10 & 1.358 & 124.792 \\
\hline 1991AP-5.5 & Stanley/Mt. Pleasant Airfield & UK (Falkland Islands) & -51.82 & -58.45 & 1247 & 1991/08/12-15 & 5 & Hudson & $1508-057$ & -45.90 & -72.97 \\
\hline 1991AP-5.4 & Mar del Plata/ Mar del Plata AP & Argentina & -37.933 & -57.567 & 1548 & 1991/08/12-15 & 5 & Hudson & $1508-057$ & -45.90 & -72.97 \\
\hline 1991AP-5.3 & Puerto Julian/San Julian AP & Argentina & -49.25 & -67.8 & 537 & 1991/08/12-15 & 5 & Hudson & $1508-057$ & -45.90 & -72.97 \\
\hline 1991AP-5.2 & Puerto Deseado/Puerto Deseado AP & Argentina & -47.717 & -65.917 & 573 & 1991/08/12-15 & 5 & Hudson & $1508-057$ & -45.90 & -72.97 \\
\hline 1991AP-5.1 & Comodoro Rivadavia/C. Rivadavia AP & Argentina & -45.783 & -67.467 & 427 & 1991/08/12-15 & 5 & Hudson & $1508-057$ & -45.90 & -72.97 \\
\hline 1991AP-4.1 & Manila/Ninoy Aquino Intl AP & Philippines & 14.517 & 121.017 & 99 & 1991/07/17 & 6 & Pinatubo & 0703-083 & 15.13 & 120.35 \\
\hline 1991AP-3.7 & Manila/Ninoy Aquino Intl AP & Philippines & 14.517 & 121.017 & 99 & 1991/06/12-15 & 6 & Pinatubo & 0703-083 & 15.13 & 120.35 \\
\hline 1991AP-3.6 & Sangley Point Air Base & Philippines & 14.5 & 120.9 & 92 & 1991/06/12-15 & 6 & Pinatubo & 0703-083 & 15.13 & 120.35 \\
\hline 1991AP-3.5 & Cubi Point Naval Air Station & Philippines (US base) & 14.78 & 120.27 & 40 & 1991/06/12-15 & 6 & Pinatubo & 0703-083 & 15.13 & 120.35 \\
\hline 1991AP-3.4 & Clark Air Base & Philippines (US base) & 15.183 & 120.533 & 21 & 1991/06/12-15 & 6 & Pinatubo & 0703-083 & 15.13 & 120.35 \\
\hline 1991AP-3.3 & Basa Air Base & Philippines & 15 & 120.5 & 22 & 1991/06/12-15 & 6 & Pinatubo & 0703-083 & 15.13 & 120.35 \\
\hline 1991AP-3.2 & Legaspi/Legaspi AP & Philippines & 13.167 & 123.733 & 425 & 1991/06/12-15 & 6 & Pinatubo & 0703-083 & 15.13 & 120.35 \\
\hline 1991AP-3.1 & Puerto Princesa/Puerto Princesa AP & Philippines & 9.733 & 118.75 & 625 & 1991/06/12-15 & 6 & Pinatubo & 0703-083 & 15.13 & 120.35 \\
\hline 1991AP-2.1 & Kumamoto/Kumamoto AP & Japan & 32.8 & 130.77 & 44 & 1991/06/03 & 1 & Unzen & 0802-10 & 32.75 & 130.30 \\
\hline 1991AP-1.1 & Colima/Colima AP & Mexico & 19.3 & -103.667 & 24 & $1991 / 04 / 16$ & 2 & Colima & $1401-04$ & 19.514 & -103.62 \\
\hline 1990AP-3.1 & Kagoshima/Kagoshima AP & Japan & 31.55 & 130.567 & 10 & 1990/03/11 & 3 & Sakurajima & 0802-08 & 31.58 & 130.67 \\
\hline 1990AP-2.1 & Anchorage/Anchorage Intl AP & USA & 61.167 & -149.983 & 168 & 1990/02/21,28 & 3 & Redoubt & $1103-03$ & 60.48 & -152.75 \\
\hline 1990AP-1.1 & Kenai/Kenai Municipal AP & USA & 60.583 & -152.15 & 35 & 1990/01/08 & 3 & Redoubt & $1103-03$ & 60.48 & -152.75 \\
\hline 1989AP-1.1 & Anchorage/Anchorage Intl AP & USA & 61.167 & -149.983 & 168 & 1989/12/15 & 3 & Redoubt & 1103-03 & 60.48 & -152.75 \\
\hline 1986AP-2.1 & Catania/Fontanarossa Intl AP & Italy & 37.467 & 15.067 & 30 & 1986/09/24 & 2 & Etna & 0101-06 & 37.734 & 15.004 \\
\hline 1986AP-1.1 & Anchorage/Anchorage Intl AP & USA & 61.167 & -149.983 & 275 & 1986/03/27-31 & 4 & Augustine & 1103-01 & 59.37 & -153.42 \\
\hline 1984AP-2.1 & Legaspi/Legaspi AP & Philippines & 13.167 & 123.733 & 11 & 1984/09/23-24 & 3 & Mayon & 0703-03 & 13.257 & 123.685 \\
\hline 1984AP-1.2 & Gorontola/Tolotio AP & Indonesia & 0.65 & 122.917 & 207 & $1984 / 05 / 24-26$ & 3 & Soputan & 0606-03 & 1.108 & 124.725 \\
\hline 1984AP-1.1 & Manado/Sam Ratulangi AP & Indonesia & 1.533 & 124.917 & 52 & 1984/05/24-26 & 3 & Soputan & 0606-03 & 1.108 & 124.725 \\
\hline 1983AP-2.1 & Miyakejima/Miyakejima AP & Japan & 34.067 & 139.567 & 4 & 1983/10/03 & 3 & Miyake-jima & 0804-04 & 34.08 & 139.53 \\
\hline 1983AP-1.1 & AP west of Gamalama & Indonesia & unknown & unknown & & 1983/08/09 & 3 & Gamalama & 0608-06 & 0.80 & 127.325 \\
\hline 1982AP-2.4 & Bandung/Husein Sastranegara AP & Indonesia & -6.901 & 107.577 & 65 & 1982/04/5-1983/01/08 & 4 & Galunggung & 0603-14 & -7.25 & 108.05 \\
\hline 1982AP-2.3 & Bandung/Husein Sastranegara AP & Indonesia & -6.901 & 107.577 & 65 & 1982/04/5-1983/01/08 & 4 & Galunggung & 0603-14 & -7.25 & 108.05 \\
\hline 1982AP-2.2 & Bandung/Husein Sastranegara AP & Indonesia & -6.901 & 107.577 & 65 & 1982/04/5-1983/01/08 & 4 & Galunggung & 0603-14 & -7.25 & 108.05 \\
\hline 1982AP-2.1 & Bandung/Husein Sastranegara AP & Indonesia & -6.901 & 107.577 & 65 & 1982/04/5-1983/01/08 & 4 & Galunggung & 0603-14 & -7.25 & 108.05 \\
\hline 1982AP-1.2 & Tuxtla Gutierrez/Lano San Juan AP & Mexico & 16.75 & -93.12 & 69 & $1982 / 04 / 03$ & 5 & E Chichon & 1401-12 & 17.360 & -93.228 \\
\hline 1982AP-1.1 & Villahermosa/C.R.Rovirosa Perez Intl AP & Mexico & 17.99 & -92.98 & 75 & 1982/04/03 & 5 & $\boxminus$ Chichon & $1401-12$ & 17.360 & -93.228 \\
\hline
\end{tabular}


Table 1. Compilation of Disruptions to Airports by Volcanic Activity (Version 1.0, 1944-2006)

By M. Guffanti, G.C. Mayberry, T.J. Casadevall, R. Wunderman. Data fields explained in report text. (AP=Airport)

\begin{tabular}{|c|c|c|c|c|c|c|c|c|c|c|c|}
\hline Incident \# & CitylAirport & Country1 & APLat & APLong & Dist, km & Erupt Date Y/MVD & $\mathbf{V E}$ & Volcano & VNum & VLat & VLong \\
\hline 1980AP-3.1 & Portland/Portland Intl AP & USA & 45.583 & -122.6 & 76 & $1980 / 06 / 12$ & 5 & St. Helens & $1201-05$ & 46.20 & -122.18 \\
\hline 1980AP-2.1 & Portland/Portland Intl AP & USA & 45.583 & -122.6 & 76 & $1980 / 05 / 25$ & 5 & St. Helens & 1201-05 & 46.20 & -122.18 \\
\hline 1980AP-1.6 & Spokane/Fairchild AFB & USA & 47.626 & -117.644 & 351 & 1980/05/18 & 5 & St. Helens & $1201-05$ & 46.20 & -122.18 \\
\hline 1980AP-1.5 & Spokane/Spokane Intl AP & USA & 47.633 & -117.533 & 387 & 1980/05/18 & 5 & St. Helens & 1201-05 & 46.20 & -122.18 \\
\hline 1980AP-1.4 & Moses Lake/Grant County AP & USA & 47.095 & -119.261 & 244 & 1980/05/18 & 5 & St. Helens & 1201-05 & 46.20 & -122.18 \\
\hline 1980AP-1.3 & Yakima/Yakima Air Terminal & USA & 46.567 & -120.533 & 133 & 1980/05/18 & 5 & St. Helens & 1201-05 & 46.20 & -122.18 \\
\hline 1980AP-1.2 & Pullman/Pullman Moscow Regional AP & USA & 46.75 & -117.117 & 393 & 1980/05/18 & 5 & St. Helens & $1201-05$ & 46.20 & -122.18 \\
\hline 1980AP-1.1 & Missoula/Missoula Intl AP & USA & 46.917 & -114.083 & 624 & 1980/05/18 & 5 & St. Helens & 1201-05 & 46.20 & -122.18 \\
\hline 1979AP-1.1 & Catania/Fontanarossa Intl AP & Italy & 37.467 & 15.067 & 30 & 1979/08/03 & 2 & Etna & 0101-06 & 37.734 & 15.004 \\
\hline 1976AP-1.1 & Anchorage/Anchorage Intl AP & USA & 61.167 & -149.983 & 275 & 1976/01/23-25 & 4 & Augustine & 1103-01 & 59.37 & -153.42 \\
\hline 1971AP-1.1 & Kagoshima/Kagoshimna AP & Japan & 31.55 & 130.567 & 10 & 1971/09/09 & 3 & Sakurajima & 0802-08 & 31.58 & 130.67 \\
\hline 1963AP-2.1 & Surabaya/Surabaya-Juanda Intl AP & Indonesia & -7.217 & 112.717 & 1758 & 1963/03/17 & 5 & Agung & 0604-02 & 8.342 & 115.508 \\
\hline 1963AP-1.1 & San Jose/Juan Santamaria Intl AP & Costa Rica & 9.93 & -84.1 & 28 & 1963/03/13 & 3 & Irazu & 1405-06 & 9.979 & -83.852 \\
\hline 1957AP-1.1 & Iwo-jima/Iwo-jima Airfield & Japan & 24.784 & 141.323 & 4 & 1957/03/28 & 1 & Iwo-jima & 0804-12 & 24.754 & 141.29 \\
\hline 1953AP-1.3 & Anchorage/Merrill Field & USA & 61.2 & -149.833 & 130 & 1953/07/09 & 4 & Spurr & $1103-04$ & 61.30 & -152.25 \\
\hline 1953AP-1.2 & Anchorage/Emendorf AFB & USA & 61.253 & -149.792 & 131 & 1953/07/09 & 4 & Spurr & 1103-04 & 61.30 & -152.25 \\
\hline 1953AP-1.1 & Anchorage/Anchorage Intl AP & USA & 61.167 & -149.983 & 122 & 1953/07/09 & 4 & Spurr & $1103-04$ & 61.30 & -152.25 \\
\hline 1951AP-1.1 & Port Moresby/Jacksons AP & Papua New Guinea & -9.441 & 147.220 & 116 & $1951 / 01 / 21$ & 4 & Lamington & 0503-01 & -8.95 & 148.15 \\
\hline 1944AP-1.1 & Naples & Italy & 40.883 & 14.283 & 14 & 1944/03/22 & 3 & Vesunius & 0101-02 & 40.821 & 14.426 \\
\hline
\end{tabular}


Table 1. Compilation of Disruptions to Airports by Volcanic Activity (Version 1.0, 1944-2006)

By M. Guffanti, G.C. Mayberry, T.J. Casadevall, R. Wunderman. Data fields explained in report text. (AP=Airport)

\begin{tabular}{|c|c|c|c|c|}
\hline Incident \# & Country2 & Hazard & Days closed & References \\
\hline 2006AP-5.2 & Italy & AA & 14 nights & C. Holliday pers. comm. 2006 \\
\hline 2006AP-5.1 & Italy & AA & 14 nights & Agenzia Giornalistica Italia 6Dec2006 \\
\hline 2006AP-4.1 & Papua New Guinea & AF & 4 & Austalian Broadcasting Corp 110ct2006 \\
\hline 2006AP-3.6 & Ecuador & $A F$ & 2 & C. Holliday pers. comm. 2006 \\
\hline 2006AP-3.5 & Ecuador & AF & $?$ & Diario del Hoy 17July2006 \\
\hline 2006AP-3.4 & Ecuador & AA? & $?$ & Diario del Hoy 17July2006 \\
\hline 2006AP-3.3 & Ecuador & AF & $?$ & Đ Comercio 18July2006 \\
\hline 2006AP-3.2 & Ecuador & AA & 0 & Diario del Hoy 17July2006 \\
\hline 2006AP-3.1 & Ecuador & AF & $?$ & Diario del Hoy 17July2006 \\
\hline 2006AP-2.5 & UK (Montserrat) & $A A$ & 0 & Associated Press 22May2006 \\
\hline 2006AP-2.4 & UK (Montserrat) & AA & 0 & Associated Press 22May2006 \\
\hline 2006AP-2.3 & UK (Montserrat) & $A A$ & 0 & Associated Press 22May2006 \\
\hline 2006AP-2.2 & UK (Montserrat) & $A A$ & 0 & Associated Press 22May2006 \\
\hline 2006AP-2.1 & UK (Montserrat) & AA & 0 & Associated Press 22May2006 \\
\hline 2006AP-1.1 & USA & AA & 0 & C. Neal pers. comm 2006 \\
\hline 2005AP-9.1 & Colombia & AF & 0 & E Tiempo 24Nov2005 \\
\hline 2005AP-8.1 & USA (CNMI) & AA & 4 & Saipan Tribune 8Aug2005; Pacific Daily News 2Aug2005 \\
\hline 2005AP-7.1 & Mexico & AF & $?$ & LATimes Wre Reports 31May2005 \\
\hline 2005AP-6.1 & Philippines & AA & 0 & Inquirer News Service 3May2005, BGVN 2005 (30:06) \\
\hline 2005AP-5.1 & Comoros & AF & $3 ?$ & Reuters 17April2005; BGVN 2005 (30:04) \\
\hline 2005AP-4.1 & USA (CNMI) & AA & $?$ & Saipan Tribune 8Aug2005; Pacific Daily News 2Aug2005, BGVN 2005 (30:04) \\
\hline 2005AP-3.1 & Papua New Guinea & AF,AA & $?$ & BGVN 2005 (30:07); Post Courier 2Mar2005 \\
\hline 2005AP-2.1 & USA (CNMI) & AA & 0 & Saipan Tribune 15Feb2005, BGVN 2005 (30:02) \\
\hline 2005AP-1.1 & Papua New Guinea & AA & $?$ & BGVN2005 (30:05) \\
\hline $2004 A P-6.1$ & Iceland & AA? & $1 ?$ & S. Karlsdottir pers. comm. 2006; Times Online 5Nov2004 \\
\hline $2004 A P-5.1$ & Papua New Guinea & AF,AA & 0 & BGVN 2004 (29:10); D. Innes pers. cmm. 2006 \\
\hline $2004 A P-4.1$ & Japan & AF & 0 & Star\&Stripes 21Sept2004; AFWA Alert 16Sept2004 \\
\hline 2004AP-3.1 & Indonesia & AF & 1 & Jakarta Post 15Sept2004 \\
\hline 2004AP-2.1 & Indonesia & AF & $>2$ & Reuters 8June2004; VSI written comm 2004, BGVN 2004 (29:05) \\
\hline 2004AP-1.1 & Russia & AF & 1 & BGVN 2004 ( 29:05) \\
\hline 2003AP-5.4 & Ecuador & AA & 0 & E Comercio 29Aug2003 \\
\hline 2003AP-5.3 & Ecuador & AA & 0 & 巨 Comercio 29Aug2003 \\
\hline 2003AP-5.2 & Ecuador & AF & 0 & BGVN 2003 (28:11) \\
\hline 2003AP-5.1 & Ecuador & AA & 0 & E Comercio 29Aug2003 \\
\hline 2003AP-4.7 & UK (Montserrat) & AF & 0 & Associated Press 13July2003 \\
\hline 2003AP-4.6 & UK (Montserrat) & AF & 0 & Associated Press 13July2003 \\
\hline 2003AP-4.5 & UK (Montserrat) & $A F$ & 0 & Associated Press 13July2003 \\
\hline 2003AP-4.4 & UK (Montserrat) & $A F$ & 0 & Associated Press 13July2003 \\
\hline
\end{tabular}


Table 1. Compilation of Disruptions to Airports by Volcanic Activity (Version 1.0, 1944-2006)

By M. Guffanti, G.C. Mayberry, T.J. Casadevall, R. Wunderman. Data fields explained in report text. (AP=Airport)

\begin{tabular}{|c|c|c|c|c|}
\hline Incident \# & Country 2 & Hazard & Days close & References \\
\hline 2003AP-4.3 & UK (Montserrat) & AF & 0 & Associated Press 13July2003 \\
\hline 2003AP-4.2 & UK (Montserrat) & $A F$ & 0 & Associated Press 13July2003 \\
\hline 2003AP-4.1 & UK (Montserrat) & AF & 0 & Associated Press 13July2003 \\
\hline 2003AP-3.2 & USA (CNMI) & AF,AA & 0 & Saipan Tribune 24May03; BGVN 2003 (28:05) \\
\hline 2003AP-3.1 & USA (CNMI) & AF,AA & 0 & Saipan Tribune 24May2003; BGVN 2003 (28:05) \\
\hline 2003AP-2.1 & UK (Montserrat) & AA? & 0 & L. Salinas pers. comm 2003 \\
\hline 2003AP-1.1 & Papua New Guinea & AF & $?$ & BGVN 2003 (28:03) \\
\hline 2002AP-6.1 & Ecuador & AF & 8 & Reuters 3Nov2002; BGVN 2002 (27:11) \\
\hline 2002AP-5.3 & Italy & AF & many & BGVN 2002 (27:11, 27:12); Coltelli 2006 \\
\hline 2002AP-5.2 & Italy & AF & many & BGVN 2002 (27:11); Coltelli 2006 \\
\hline 2002AP-5.1 & Italy & AF & $?$ & S. Eckhert pers. comm. 2002 \\
\hline 2002AP-4.1 & Papua New Guinea & AA & 6 & BGVN $2002(27: 11)$ \\
\hline 2002AP-3.1 & UK (Montserrat) & AF,AA & 0 & C. Holliday pers. comm 2002 \\
\hline 2002AP-2.1 & Papua New Guinea & AF & $?$ & Reuters 9Aug2002; David Innes pers. comm. 2003 \\
\hline 2002AP-1.1 & D.R. Congo & LF & $?$ & BGVN 2001 (26:12), 2002 (27:04) \\
\hline 2001AP-5.4 & UK (Montserrat) & AF & 0 & Reuters 31July2001; BGVN 2001 (26:07) \\
\hline 2001AP-5.3 & UK (Montserrat) & $A F$ & 0 & Reuters 31July2001; BGVN 2001 (26:07) \\
\hline 2001AP-5.2 & UK (Montserrat) & AF & 0 & Reuters 31July2001; BGVN 2001 (26:07) \\
\hline 2001AP-5.1 & UK (Montserrat) & AF & 0 & Reuters 31July2001; BGVN 2001 (26:07) \\
\hline 2001AP-4.1 & Italy & $A F$ & $?$ & BGVN 2001 (26:09); BGVN 2002 (27:03) \\
\hline 2001AP-3.1 & Mexico & AF & 0 & BGVN 2001 ( 26:08) \\
\hline 2001AP-2.1 & Philippines & AF & 11 & Agence France Presse 3July2001 \\
\hline 2001AP-1.1 & Indonesia & AF & $?$ & BGVN 2001 (26:11) \\
\hline 2000AP-6.1 & Japan & AF & 0 & BGVN 2000 (25:07); Tupper et al. 2004 \\
\hline 2000AP-5.1 & New Zealand & AF,AA & $?$ & BGVN 2000 ( 25:07); Johnston et al. 2000 \\
\hline 2000AP-4.2 & Italy & $A F$ & $?$ & BGVN 2000 (25:06) \\
\hline 2000AP-4.1 & Italy & AF & $?$ & BGVN 2000 (25:06) \\
\hline 2000AP-3.1 & Philippines & AA & 1 & Airline Industry Information 25Feb2000 \\
\hline 2000AP-2.2 & Ecuador & AF & $?$ & BGVN 2000 (25:04) \\
\hline 2000AP-2.1 & Ecuador & AF & $?$ & BGVN 2000 (25:04) \\
\hline 2000AP-1.1 & Guatemala & AF & 0.5 & Prensa Libre 17Jan2000 \\
\hline 1999AP-3.1 & Ecuador & $A F$ & $?$ & BGVN $1999(24: 10)$ \\
\hline 1999AP-2.1 & Ecuador & AF & 3 & BGVN 1999 (24:09); J. Ewert pers. comm. 2002 \\
\hline 1999AP-1.1 & Guatemala & AF,AA & $?$ & BGVN 2002 (27:03) \\
\hline 1998AP-4.1 & Mexico & AF & $<1$ & BGVN 1998 ( 23:12) \\
\hline 1998AP-3.1 & Guatemala & $A F$ & 2 & BGVN 1999 (24:02) \\
\hline 1998AP-2.1 & Italy & AF & $<1$ & BGVN 1998 (23:11) \\
\hline 1998AP-1.1 & Guatemala & AF & 3 & BGVN 1998 (23:05) \\
\hline
\end{tabular}


Table 1. Compilation of Disruptions to Airports by Volcanic Activity (Version 1.0, 1944-2006)

By M. Guffanti, G.C. Mayberry, T.J. Casadevall, R. Wunderman. Data fields explained in report text. (AP=Airport)

\begin{tabular}{|c|c|c|c|c|}
\hline Incident \# & Country2 & Hazard & Days closed & References \\
\hline 1997AP-5.1 & UK (Montserrat) & AF? & $?$ & BGVN 1997 (22:09) \\
\hline 1997AP-4.1 & Mexico & $A P$ & 0.5 & BGVN 1997 (22:07) \\
\hline 1997AP-3.1 & UK (Montserrat) & PF & permanent & BGVN 1997 ( 22:06) \\
\hline 1997AP-2.1 & UK (Montserrat) & PF & 1 & W. Aspinall pers. comm. 2003 \\
\hline 1997AP-1.1 & Papua New Guinea & AF & 1 & BGVN 1997 (22:03) \\
\hline 1996AP-3.1 & UK (Montserrat) & AA? & $<1$ & BGVN 1996 (21:09) \\
\hline 1996AP-2.1 & UK (Montserrat) & PF & $?$ & BGVN 1996 (21:09) \\
\hline 1996AP-1.11 & New Zealand & AA & 0 & Johnston et al. 2000; P. Lechner written comm. 2004 \\
\hline 1996AP-1.10 & New Zealand & AA & 0 & Johnston et al. 2000; P. Lechner written comm 2004 \\
\hline 1996AP-1.09 & New Zealand & AA & 0 & Johnston et al. 2000; P. Lechner written comm. 2004 \\
\hline 1996AP-1.08 & New Zealand & AA & 0 & Johnston et al. 2000; P. Lechner written comm. 2004 \\
\hline 1996AP-1.07 & New Zealand & AF & $?$ & Johnston et al. 2000; P. Lechner written comm 2004 \\
\hline 1996AP-1.06 & New Zealand & AF & $?$ & Johnston et al. 2000; P. Lechner written comm 2004 \\
\hline 1996AP-1.05 & New Zealand & AF & $?$ & Johnston et al. 2000; P. Lechner written comm. 2004 \\
\hline 1996AP-1.04 & New Zealand & AF & $?$ & Johnston et al. 2000; P. Lechner written comm. 2004 \\
\hline 1996AP-1.03 & New Zealand & AF & $?$ & Johnston et al. 2000; P. Lechner written comm. 2004 \\
\hline 1996AP-1.02 & New Zealand & AF & 1 & T. Hurst pers. comm. 2007; Johnston et al. 2004 \\
\hline 1996AP-1.01 & New Zealand & AF,AA & $?$ & BGVN 1996 (21:05); Johnston et al. 2000 \\
\hline 1995AP-3.1 & UK (Montserrat) & AF & $<1$ & BGVN 1995 (20:10) \\
\hline 1995AP-2.9 & New Zealand & AA & 0 & Johnston et al. 2000; P. Lechner written comm. 2004 \\
\hline 1995AP-2.8 & New Zealand & AA & 0 & Johnston et al. 2000; P. Lechner written comm. 2004 \\
\hline 1995AP-2.7 & New Zealand & AA & 0 & Johnston et al. 2000; P. Lechner written comm 2004 \\
\hline 1995AP-2.6 & New Zealand & AA & 0 & Johnston et al. 2000; P. Lechner written comm. 2004 \\
\hline 1995AP-2.5 & New Zealand & AA & 0 & Johnston et al. 2000; P. Lechner written comm 2004 \\
\hline 1995AP-2.4 & New Zealand & AA & 0 & Johnston et al. 2000; P. Lechner written comm. 2004 \\
\hline 1995AP-2.3 & New Zealand & AA & 0 & Johnston et al. 2000; P. Lechner written comm. 2004 \\
\hline 1995AP-2.2 & New Zealand & AF & $?$ & Johnston et al. 2000; P. Lechner written comm 2004 \\
\hline 1995AP-2.1 & New Zealand & AF & $?$ & Johnston et al. 2000; P. Lechner written comm 2004 \\
\hline 1995AP-1.1 & Mexico & AF & $?$ & BGVN 1995 ( 20:03) \\
\hline 1994AP-2.2 & Chile & AF & $?$ & BGVN 1994 (19:04) \\
\hline 1994AP-1.2 & Papua New Guinea & $A F, A A$ & permanent & BGVN 1994 (19:08); R. Stewart pers. comm. 2005 \\
\hline 1994AP-1.1 & Papua New Guinea & AF & $?$ & BGVN $1994(19: 08)$ \\
\hline 1993AP-2.1 & Colombia & AF & $<1$ & BGVN 1993 (18:05) \\
\hline 1993AP-1.4 & Chile & AF & $?$ & Casadevall 1993 \\
\hline 1993AP-1.3 & Chile & AF & $?$ & Casadeval, 1993 \\
\hline 1993AP-1.2 & Chile & AF & $?$ & Casadevall 1993 \\
\hline 1993AP-1.1 & Chile & AF & $?$ & Casadevall 1993 \\
\hline 1992AP-2.4 & USA & AF & $?$ & BGVN $1992(17: 07)$ \\
\hline
\end{tabular}


Table 1. Compilation of Disruptions to Airports by Volcanic Activity (Version 1.0, 1944-2006)

By M. Guffanti, G.C. Mayberry, T.J. Casadevall, R. Wunderman. Data fields explained in report text. (AP=Airport)

\begin{tabular}{|c|c|c|c|c|}
\hline Incident \# & Country2 & Hazard & Days closed & References \\
\hline 1992AP-2.3 & USA & AF & $<1$ & Casadevall 1993 \\
\hline 1992AP-2.2 & USA & AF & $?$ & Casadevall 1993 \\
\hline 1992AP-2.1 & USA & AF & 3 & Casadevall 1993 \\
\hline 1992AP-1.1 & Japan & AF & 1 & Casadevall 1993 \\
\hline 1991AP-6.2 & Indonesia & AA & "several" & Casadevall 1993 \\
\hline 1991AP-6.1 & Indonesia & AF & 5 & Casadevall 1993; BGVN 1991 (16:10) \\
\hline 1991AP-5.5 & Chile & AF,AA & $?$ & Casadevall 1993; BGVN 1991 (16:08) \\
\hline 1991AP-5.4 & Chile & AF,AA & $?$ & Casadevall 1993; BGVN 1991 (16:08) \\
\hline 1991AP-5.3 & Chile & AF,AA & $?$ & Casadevall 1993; BGVN 1991 (16:08) \\
\hline 1991AP-5.2 & Chile & AF,AA & $?$ & Casadevall 1993; BGVN 1991 (16:08) \\
\hline 1991AP-5.1 & Chile & AF,AA & $?$ & Casadevall 1993; BGVN 1991 (16:08) \\
\hline 1991AP-4.1 & Philippines & AF,AA & 2 & Casadevall et al. 1996 \\
\hline 1991AP-3.7 & Philippines & AF & 5 & Casadevall 1993; Casadevall et al. 1996 \\
\hline 1991AP-3.6 & Philippines & AF & $?$ & Casadevall 1993; Casadevall et al. 1996 \\
\hline 1991AP-3.5 & Philippines & AF & 13 & Casadevall 1993; Casadevall et al. 1996 \\
\hline 1991AP-3.4 & Philippines & AF & permanent & Casadevall 1993; Casadevall et al. 1996 \\
\hline 1991AP-3.3 & Philippines & AF & $?$ & Casadevall 1993; Casadevall et al. 1996 \\
\hline 1991AP-3.2 & Philippines & AF & $?$ & Casadevall 1993; Casadevall et al. 1996 \\
\hline 1991AP-3.1 & Philippines & AF & $?$ & Casadevall 1993; Casadevall et al. 1996 \\
\hline 1991AP-2.1 & Japan & AF,AA & 0 & Casadevall 1993 \\
\hline 1991AP-1.1 & Mexico & AF & "several" & BGVN 1991 (16:04) \\
\hline 1990AP-3.1 & Japan & AF & $<1$ & Casadevall 1993 \\
\hline 1990AP-2.1 & USA & $A F, A A$ & 0 & Casadevall 1993 \\
\hline 1990AP-1.1 & USA & AF & "several" & Casadevall 1993 \\
\hline 1989AP-1.1 & USA & AF, AA & 0 & Casadevall 1993 \\
\hline 1986AP-2.1 & Italy & AF & 2 & SEAN $1986(11: 09)$ \\
\hline 1986AP-1.1 & USA & AF & 0 & BGVN 1986 (11:03); C. Neal pers. comm. 2006 \\
\hline 1984AP-2.1 & Philippines & AA & $?$ & C. Newhall pers. comm. 2007 \\
\hline 1984AP-1.2 & Indonesia & AF & 2 & SEAN 1984 (09:05) \\
\hline 1984AP-1.1 & Indonesia & AF & 2 & SEAN 1984 (09:05) \\
\hline 1983AP-2.1 & Japan & AF & 4 & Blong 1984; SEAN 1983 (08:09, 08:10) \\
\hline 1983AP-1.1 & Indonesia & AF & $?$ & SEAN $1983(08: 07)$ \\
\hline 1982AP-2.4 & Indonesia & AF,AA & 1 & Casadevall 1993 \\
\hline 1982AP-2.3 & Indonesia & AF,AA & 1 & Casadevall 1993 \\
\hline 1982AP-2.2 & Indonesia & AF,AA & 1 & Casadevall 1993 \\
\hline 1982AP-2.1 & Indonesia & AF,AA & 18 & Casadevall 1993 \\
\hline 1982AP-1.2 & Mexico & AF & $?$ & BGVN 1982 (07:03) \\
\hline 1982AP-1.1 & Mexico & AF & $?$ & BGVN 1982 (07:03) \\
\hline
\end{tabular}


Table 1. Compilation of Disruptions to Airports by Volcanic Activity (Version 1.0, 1944-2006)

By M. Guffanti, G.C. Mayberry, T.J. Casadevall, R. Wunderman. Data fields explained in report text. (AP=Airport)

\begin{tabular}{|c|c|c|c|c|}
\hline Incident \# & Country2 & Hazard & Days close & References \\
\hline 1980AP-3.1 & USA & AF & "several" & Schuster 1983; Casadevall 1993 \\
\hline 1980AP-2.1 & USA & AF & 0 & Schuster 1983; Casadevall 1993 \\
\hline 1980AP-1.6 & USA & AF & $?$ & Fairchild AFB Fact Sheet "Fairchild Air Force Base History" \\
\hline 1980AP-1.5 & USA & AF & 3 & Schuster 1981, 1983; Casadevall 1993 \\
\hline 1980AP-1.4 & USA & AF & 15 & Warrick et al. 1981; Casadevall 1993 \\
\hline 1980AP-1.3 & USA & $A F$ & 7 & Casadevall 1993; Blong 1984 \\
\hline 1980AP-1.2 & USA & AF & 7 & Casadevall 1993 \\
\hline 1980AP-1.1 & USA & AF & "several" & Casadevall 1993; Blong 1984 \\
\hline 1979AP-1.1 & Italy & AF & $1 ?$ & Guest et al. 1980; Casadevall 1993 \\
\hline 1976AP-1.1 & USA & AF & 0 & Kienle \& Swanson 1985; Casadevall 1993; Jennings 1969 \\
\hline 1971AP-1.1 & Japan & AF & $<1$ & Casadevall 1993 \\
\hline 1963AP-2.1 & Indonesia & AF & 1 & Jennings 1969; Suryo 1981; Casadevall 1993 \\
\hline 1963AP-1.1 & Costa Rica & AF & 0 & E. Duarte pers. comm. 2006 \\
\hline 1957AP-1.1 & USA (post WMI) & EX,GD & $?$ & Comin \& Foster 1959 \\
\hline 1953AP-1.3 & USA & AF & $4-7$ & Juhle \& Coulter 1955; Wilcox 1959; Casadevall 1993; Blong 1984 \\
\hline 1953AP-1.2 & USA & AF & $4-7$ & Juhle \& Coulter 1955; Wlcox 1959; Casadevall 1993; Blong 1984 \\
\hline 1953AP-1.1 & USA & AF & $4-7$ & Juhle \& Coulter 1955; Wlcox 1959; Casadevall 1993; Blong 1984 \\
\hline 1951AP-1.1 & Papua New Guinea & AF & $?$ & Taylor 1958; Casadevall 1993; Blong 1984 \\
\hline 1944AP-1.1 & Italy & AF & $?$ & Uoyd 1990; New York Times 25Apr1944; Casadevall 1993 \\
\hline
\end{tabular}


Table 1. Compilation of Disruptions to Airports by Volcanic Activity (Version 1.0, 1944-2006)

By M. Guffanti, G.C. Mayberry, T.J. Casadevall, R. Wunderman. Data fields explained in report text. (AP=Airport)

\begin{tabular}{|c|c|}
\hline Incident \# & Comment \\
\hline 2006AP-5.2 & night time flights restricted 29 November to 8 December \& 13-16 December \\
\hline 2006AP-5.1 & AP closed 6 PM-7AM for -2 weeks from 24 November as precaution, daytime flights OK; some flights cancelled or diverted \\
\hline 2006AP-4.1 & ash covered airstrip; AP closed 7-10 October \\
\hline 2006AP-3.6 & fine dusting of ash; AP closed 17-18 July 2006 \\
\hline 2006AP-3.5 & $>3$ cmashfall; AP closed \\
\hline 2006AP-3.4 & AP closed \\
\hline 2006AP-3.3 & AP closed \\
\hline 2006AP-3.2 & flights to/from Guayaquil cancelled; alternate routes used for other destinations \\
\hline 2006AP-3.1 & AP closed \\
\hline 2006AP-2.5 & Aeropostal flights to/from Caracas/Miami grounded 21 May to/from Aruba, Curacao, Cuba, Dom. Rep; American Airlines suspended flights to/from Miami/Caracas \\
\hline 2006AP-2.4 & all fights canceled on 21st, including to Caracas, Miami, NY, San Juan, Puerto Rico, Toronto \\
\hline 2006AP-2.3 & Aeropostal flights grounded on 21st to/from Caracas, Aruba, Curacao, Cuba, \& Dominican Republic \\
\hline 2006AP-2.2 & Aeropostal flights grounded on 21st to/from Caracas, Aruba, Curacao, Cuba, \& Dominican Republic \\
\hline 2006AP-2.1 & Aeropostal flights grounded on 21st to/from Caracas, Aruba, Curacao, Cuba, \& Dominican Republic \\
\hline 2006AP-1.1 & Alaska Airlines cancelled 36 flights to/from AP on 13 Jan, FedEx routed 5 inbound planes to Fairbanks, local flights to Homer cancelled/delayed; on 17 th \\
\hline 2005AP-9.1 & 300 people stranded at airport \\
\hline 2005AP-8.1 & flights suspended 2-5 Aug; poor of visibility owing to volcanic haze; hundreds unable to depart Saipan \\
\hline 2005AP-7.1 & "A shower of ash closed the airport" \\
\hline 2005AP-6.1 & 2-km visibility; flights to/from Kalibo suspended on 3 May, affecting Boracay resort; 100's of passengers stranded; smaller carriers also grounded \\
\hline 2005AP-5.1 & ashfall on western \& northern parts of island; flights to island cancelled, resumed on 19 April \\
\hline 2005AP-4.1 & light ashfall beginning around $10 \mathrm{AM} ; \mathrm{AP}$ closed \\
\hline 2005AP-3.1 & Fine ash reached AP; many flights cancelled to/from AP during Feb \\
\hline 2005AP-2.1 & zero visibility hindered approach and landing of aircraft from Manila \\
\hline 2005AP-1.1 & 5 commercial flights cancelled from Rabaul, delaying 100 passengers \\
\hline 2004AP-6.1 & AP closed at beginning of eruption; KLM cancelled 59 flights from Amsterdam/Schiphol AP; "dozens of domestic flights N \& E of Iceland grounded by ash" \\
\hline 2004AP-5.1 & AP had only light dusting of ash, but ash haze below 10,000 ft reduced visibility \& prevented landings \\
\hline 2004AP-4.1 & trace accumulation \\
\hline 2004AP-3.1 & AP closed on 14 September; equipment \& facilities covered by up to $1 \mathrm{~cm}$ of ash \& all flights cancelled \\
\hline 2004AP-2.1 & AP closed beginning on 8 June at 0800 (for at least 2 days) \\
\hline 2004AP-1.1 & 1-2 mm of ash deposited; AP closed on 10 May \\
\hline 2003AP-5.4 & delays in schedules to/from Quito/Cuenca (Ambato also may have been affected) \\
\hline 2003AP-5.3 & delays in schedules to/from Quito/Guayaquil \\
\hline 2003AP-5.2 & ash deposition; flight restrictions to/from airport \\
\hline 2003AP-5.1 & delays in schedules to/from Quito/Guayaquil \\
\hline 2003AP-4.7 & American Airlines cancelled -50 flights from Puerto Rico to various islands; other airlines also suspended ops \\
\hline 2003AP-4.6 & American Airlines cancelled -50 flights from Puerto Rico to various islands; other airlines also suspended ops \\
\hline 2003AP-4.5 & American Airlines cancelled -50 flights from Puerto Rico to various islands; other airlines also suspended ops \\
\hline 2003AP-4.4 & American Airlines cancelled -50 flights from Puerto Rico to various islands; other airlines also suspended ops \\
\hline
\end{tabular}

Page 22 
Table 1. Compilation of Disruptions to Airports by Volcanic Activity (Version 1.0, 1944-2006)

By M. Guffanti, G.C. Mayberry, T.J. Casadevall, R. Wunderman. Data fields explained in report text. (AP=Airport)

\begin{tabular}{|c|c|}
\hline Incident \# & Comment \\
\hline 2003AP-4.3 & American Airlines cancelled -50 flights from Puerto Rico to various islands; other airlines also suspended ops \\
\hline 2003AP-4.2 & American Airlines cancelled -50 flights from Puerto Rico to various islands; other airlines also suspended ops \\
\hline 2003AP-4.1 & American Airlines cancelled -50 flights from Puerto Rico to various islands; other airlines also suspended ops \\
\hline 2003AP-3.2 & Continental Airlines cancelled 3 flights, Guam to Saipan, 23 May \\
\hline 2003AP-3.1 & Continental Airlines cancelled 3 flights, Saipan to Guam, 23 May; one of these on to Japan \\
\hline 2003AP-2.1 & landings cancelled \\
\hline 2003AP-1.1 & AP closed several times due to "ash drift " from Tavurvur \\
\hline 2002AP-6.1 & AP closed 8 days \\
\hline 2002AP-5.3 & repeated AP closures over 3 months, beginning on 27 October; inter-agency ash plan developed for future events \\
\hline 2002AP-5.2 & first of repeated AP closures beginning 31 October \\
\hline 2002AP-5.1 & Volcanic Ash Operations Plan for US Navy base written as result of 3-month-long explosive activity \\
\hline 2002AP-4.1 & Tokua AP closed 22-27 October; reopened when ash plume changed direction; 2 flights per day after reopening \\
\hline 2002AP-3.1 & only scheduled flight (C-12) cancelled \\
\hline 2002AP-2.1 & AP closed due to ashfall \\
\hline 2002AP-1.1 & lava covered N end of runway, Russian plane (possibly 2) damaged when landing (ran into solidified flow) \\
\hline 2001AP-5.4 & ash on runways; flight cancellations \\
\hline $2001 \mathrm{AP}-5.3$ & ash on runways; flight cancellations \\
\hline 2001AP-5.2 & ash on runways; flight cancellations \\
\hline 2001AP-5.1 & ash on runways; flight cancellations \\
\hline 2001AP-4.1 & AP "paralyzed" \\
\hline 2001AP-3.1 & very light ashfall on runways; some takeoffs briefly suspended \\
\hline 2001AP-2.1 & light ashfall; AP closed 23 June-3 July \\
\hline 2001AP-1.1 & Sam Ratulangi \& Cengkareng APs contacted by Volc. Survey of Indonesia; actual impacts unknown \\
\hline 2000AP-6.1 & -4 mm ash at AP; at least 2 damaging aircraft encounters with ash cloud enroute to Tokyo's Narita AP \\
\hline 2000AP-5.1 & ashfall at AP; landings \& departs restricted; Bay of Plenty traffic disrupted \\
\hline 2000AP-4.2 & sky darkened by ash; difficulties for air traffic to/from Fontanarossa AP and Sigonella NAS \\
\hline 2000AP-4.1 & sky darkened by ash; difficulties for air traffic to/from Fontanarossa AP and Sigonella NAS \\
\hline 2000AP-3.1 & all flights to AP cancelled on 24 Feb. \\
\hline 2000AP-2.2 & ashfall closed AP; also in Ambato \\
\hline 2000AP-2.1 & ashfall closed AP; also in Riobamba \\
\hline 2000AP-1.1 & AP closed from 1915 on 16th until 0600 on 17th ( -11 hours) \\
\hline 1999AP-3.1 & trace amt tephra; AP closure. Tourist sector lost $\$ 1.5 \mathrm{~N}$ day due to Oct-Nov closures \\
\hline 1999AP-2.1 & $<3$ mm ashfall; AP closed 3 days; international flights diverted to Guayaquil for few weeks \\
\hline 1999AP-1.1 & ash on runway (perhaps from Pacaya); 2 in-flight encounters with Fuego plume near airport \\
\hline 1998AP-4.1 & AP closed $2330-0115$ \\
\hline 1998AP-3.1 & AP closed for 35 hours due to fine ashfall \\
\hline 1998AP-2.1 & AP closed $15 \mathrm{~h}$ due to $\sim 1 \mathrm{~mm}$ sand-sized tephra, 1st closure since 1986 \\
\hline 1998AP-1.1 & - 2 mm tephra at AP $23 \mathrm{~N}$ of volcano; AP closed 3 days; tephra damaged aircraft on final approach \\
\hline
\end{tabular}

Page 23 
Table 1. Compilation of Disruptions to Airports by Volcanic Activity (Version 1.0, 1944-2006)

By M. Guffanti, G.C. Mayberry, T.J. Casadevall, R. Wunderman. Data fields explained in report text. (AP=Airport)

\begin{tabular}{|c|c|}
\hline Incident \# & Comment \\
\hline 1997AP-5.1 & ash drifted unusual direction (NE) affecting Antigua AP \\
\hline 1997AP-4.1 & AP closed $\sim 12 \mathrm{hr}$ while runways cleared of ash \\
\hline 1997AP-3.1 & AP closed when pyroclastic flows reached edge of airport \\
\hline 1997AP-2.1 & AP evacuated \& closed for day of 17 June \\
\hline 1997AP-1.1 & AP closed most of day due to ashfall threat \\
\hline 1996AP-3.1 & ash column to $12 \mathrm{~km}$; AP closed AM of 18th \\
\hline 1996AP-2.1 & ash from pyroclastic flows fell around island, including at AP \\
\hline 1996AP-1.11 & airspace closure \\
\hline 1996AP-1.10 & airspace closure \\
\hline 1996AP-1.09 & airspace closure \\
\hline 1996AP-1.08 & airspace closure \\
\hline 1996AP-1.07 & ashfall at $\mathrm{AP}$ \\
\hline 1996AP-1.06 & ashfall at AP \\
\hline 1996AP-1.05 & ashfall at AP \\
\hline 1996AP-1.04 & ashfall at AP \\
\hline 1996AP-1.03 & ashfall at AP \\
\hline 1996AP-1.02 & 1 mmashfall; AP closed 17 June \\
\hline 1996AP-1.01 & closures of airspace around Auckland AP \& all of North Island \\
\hline 1995AP-3.1 & ashfall closed AP for first time, briefly \\
\hline 1995AP-2.9 & airspace closure \\
\hline 1995AP-2.8 & airspace closure \\
\hline 1995AP-2.7 & airspace closure \\
\hline 1995AP-2.6 & airspace closure \\
\hline 1995AP-2.5 & airspace closure \\
\hline 1995AP-2.4 & airspace closure \\
\hline 1995AP-2.3 & airspace closure \\
\hline 1995AP-2.2 & ashfall at AP \\
\hline 1995AP-2.1 & ashfall at AP \\
\hline 1995AP-1.1 & planes dusted by ashfall \\
\hline 1994AP-2.2 & light ashfall \\
\hline 1994AP-1.2 & AP buried under $50 \mathrm{~cm}$ tephral; airspace within $110 \mathrm{~km}$ radius of AP closed; flights to/from New Britain suspended; AP permanently closed and relocated to Tokua \\
\hline 1994AP-1.1 & flights to/from New Ireland suspended; relief flights routed to Tokua AP \\
\hline 1993AP-2.1 & trace amount ashfall; closed $<1$ day \\
\hline 1993AP-1.4 & ashfall on airport, flights temporarily suspended \\
\hline 1993AP-1.3 & ashfall on airport, flights temporarily suspended \\
\hline 1993AP-1.2 & ashfall on airport, flights temporarily suspended \\
\hline 1993AP-1.1 & ashfall on airport, flights temporarily suspended \\
\hline 1992AP-2.4 & flights to/from AP halted \\
\hline
\end{tabular}


Table 1. Compilation of Disruptions to Airports by Volcanic Activity (Version 1.0, 1944-2006)

By M. Guffanti, G.C. Mayberry, T.J. Casadevall, R. Wunderman. Data fields explained in report text. (AP=Airport)

\begin{tabular}{|c|c|}
\hline Incident \# & Comment \\
\hline 1992AP-2.3 & by mid-morning on 19th field became operational; cleanup costs $-\$ 53,000$ \\
\hline 1992AP-2.2 & forewarning call from AVO; 28 aircraft evacuated, 70 aircraft shletered in hangars, 6 aircraft sealed; cleanup costs $-\$ 150,000$ \\
\hline 1992AP-2.1 & $<5$ mm ashfall; AP closed 3 days; cleanup hampered by wind redistribution of ash; cleanup costs $-\$ 250,000$ \\
\hline 1992AP-1.1 & trace amount ashfall; AP closed 1 day \\
\hline 1991AP-6.2 & no ashfall but airspace of Medan air traffic region heavily contaminated with ash; AP closed several days \\
\hline 1991AP-6.1 & AP closed till 29 October due to poor visibility and minor ashfall \\
\hline 1991AP-5.5 & light ashfall \\
\hline 1991AP-5.4 & resuspended ash for months after eruption caused reduced visibility and flight cancellations \\
\hline 1991AP-5.3 & resuspended ash for months after eruption caused reduced visibility and flight cancellations \\
\hline 1991AP-5.2 & resuspended ash for months after eruption caused reduced visibility and flight cancellations \\
\hline 1991AP-5.1 & resuspended ash for months after eruption caused reduced visibility and flight cancellations \\
\hline 1991AP-4.1 & AP closed 17-18 July; slippery runway \\
\hline 1991AP-3.7 & 0.5-1 cm ashfall; AP closed 15-19 June, resumed fully normal operations on 4 July \\
\hline 1991AP-3.6 & 0.5-1 cm ashfall \\
\hline 1991AP-3.5 & 15-20 cm sand-sized ash; AP closed on 14th, back in partial operation by 26th; parked airplanes damaged by wet ash accumulation \\
\hline 1991AP-3.4 & 15-20 cm sand-sized ash; aircraft moved in advance of eruption as result of volcanologists' forewarning \\
\hline 1991AP-3.3 & 15-20 cm sand-sized ash \\
\hline 1991AP-3.2 & trace amount ashfall \\
\hline 1991AP-3.1 & trace amount ashfall \\
\hline 1991AP-2.1 & departing airplane damaged taking off into ash cloud \\
\hline 1991AP-1.1 & $<5$ mm ashfall; AP closed several days \\
\hline 1990AP-3.1 & 2 mmashfall; AP closed $<1$ day \\
\hline 1990AP-2.1 & trace amount ashfall; no AP closure, many cancellations; in-flight encounters \\
\hline 1990AP-1.1 & -5 mm ashfall; AP closed several days \\
\hline 1989AP-1.1 & trace amount ashfall; no closure, many cancellations; damaged aircraft near Anchorage AP \\
\hline 1986AP-2.1 & lapilli \& light ash; AP closed night of 24th to morning of 25th \\
\hline 1986AP-1.1 & "dusting" in Anchorage area; USAF moved planes from Anch to Fbx; most carriers diverted/cancelled flights to/from Anch on 27-29 Mar but AP not closed \\
\hline 1984AP-2.1 & more than 73,000 people evacuated from around volcano; alert level 5 \\
\hline 1984AP-1.2 & AP closed on 26-27 May \\
\hline 1984AP-1.1 & AP closed on 26-27 May \\
\hline 1983AP-2.1 & $10 \mathrm{~cm}$ ash \& fist-sized tephra on runway; rescue planes unable to land; AP closed for 4 days \\
\hline 1983AP-1.1 & ashfall closed unnamed airport W of Gamalama \\
\hline 1982AP-2.4 & AP closed 2 September oving to reduced visibility associated with ashfall \\
\hline 1982AP-2.3 & AP closed 3 August oning to reduced visibility associated with ashfall \\
\hline 1982AP-2.2 & AP closed 14 July oving to reduced visibility associated with ashfall \\
\hline 1982AP-2.1 & AP closed 4-21 June oning to reduced visibility associated with ashfall \\
\hline 1982AP-1.2 & heavy ashfall; AP closed, roads impassable \\
\hline 1982AP-1.1 & heavy ashfall; AP closed, roads impassable \\
\hline
\end{tabular}


Table 1. Compilation of Disruptions to Airports by Volcanic Activity (Version 1.0, 1944-2006)

By M. Guffanti, G.C. Mayberry, T.J. Casadevall, R. Wunderman. Data fields explained in report text. (AP=Airport)

\begin{tabular}{|c|c|}
\hline Incident \# & Comment \\
\hline 1980AP-3.1 & 2-3 mm ashfall; AP closed several days, some carriers continued operations \\
\hline 1980AP-2.1 & $<1$ mm ashfall; no closure; flight cancellations \\
\hline 1980AP-1.6 & eruption occurred during Open House celebration, 92nd Bombardment Wing "paralyzed for 1 month" \\
\hline 1980AP-1.5 & $0.5-1 \mathrm{~cm}$ ashfall; AP closed 3 days \\
\hline 1980AP-1.4 & 7-9 cm ashfall; AP closed 15 days \\
\hline 1980AP-1.3 & 0.5-1 cm ashfall; AP closed 7 days \\
\hline 1980AP-1.2 & 1 cm ashfall; AP closed 7 days \\
\hline 1980AP-1.1 & 1-2 cm ashfall; AP closed several days \\
\hline 1979AP-1.1 & trace amount ashfall; AP closed 1 day? \\
\hline 1976AP-1.1 & trace amount ashfall; no closure, some cancellations \\
\hline 1971AP-1.1 & 3 mm ash; reduced visibility; AP closed $<1$ day \\
\hline 1963AP-2.1 & $<10$ mm ashfall; AP closed 1 day \\
\hline 1963AP-1.1 & few mm ashfall; flights re-routed to Panama or Limon \\
\hline 1957AP-1.1 & phreatic explosion blew crater in tarmac; runways repeatedly cut by faults before and since 1957 \\
\hline 1953AP-1.3 & 3-6 mm ashfall; military airfield closed $4-7$ days \\
\hline 1953AP-1.2 & 3-6 mm ashfall; military airfield closed $4-7$ days \\
\hline 1953AP-1.1 & 3-6 mm ashfall; AP closed closed 4-7 days \\
\hline 1951AP-1.1 & 1-2 mm ashfall; airfield closed \\
\hline 1944AP-1.1 & first instance of damage from an eruption to modern aircraft and facilities; 88 US military planes damaged \\
\hline
\end{tabular}

